\title{
Effect of Titanium Addition and Cooling Rate on Primary a(Al) Grains and Tensile Properties of Al-Cu Alloy
}

\author{
M. Górny and G. Sikora
}

\author{
(Submitted October 23, 2014; in revised form December 18, 2014; published online January 1, 2015)
}

\begin{abstract}
The research was conducted for castings with 3-25-mm wall thicknesses and using AITi5B1 master alloy as well as AITi75 additions. A thermal analysis was performed to determine the real cooling rate and the degree of undercooling $\left(\Delta T_{\alpha}=T_{\alpha}-T_{\min }\right.$, where $T_{\alpha}$ - the equilibrium solidification temperature, $T_{\min }$ - the minimum temperature at the beginning of $\alpha(\mathrm{Al})$ solidification) of an $\mathrm{Al}-\mathrm{Cu}$ alloy. It was shown that the Ti-based grain-refining process does not influence the cooling rate. Metallographic examinations were carried out to determine the primary grains (the number of primary $\alpha(\mathrm{Al})$ grains per unit volume). It was found that the grain count can be properly described by the exponential function of the undercooling for castings processed under various cooling and metallurgical conditions. Combining the cooling rate and the efficient grain refiner increased the tensile ductility by up to $150 \%$.
\end{abstract}

Keywords aluminum alloy, cooling rate, grain refinement, tensile properties, titanium addition

\section{Introduction}

Primary grain count is one of the most important factors for determining the quality of castings. The final number of primary $\alpha(\mathrm{Al})$ grains in the casting depends on the process of nucleation at the beginning of solidification because each nucleus gives rise to one single grain. Consequently, it represents the nucleation potential which depends on the following factors: the type and quantity of the grain refiner addition, the time and liquid metal temperature, slag and furnace atmosphere, molten metal mixing, or the chemical composition of the alloy (Ref 1-4). Not all sites found in liquid metal are involved in the nucleation process. It is estimated (Ref 5-7) that only a small percentage of them take part in the process of $\alpha(\mathrm{Al})$ phase nucleation. More sites found in the liquid metal can be activated by raising the undercooling degree. For a given nucleation potential of liquid metal, the undercooling degree can be raised by accelerating the cooling rate, which is strictly connected with the thickness of casting walls as well as the type of mold material and the pouring temperature. The cooling rate represents the thermal conditions (of heat exchange) at the beginning of solidification which in turn determine the final number of $\alpha(\mathrm{Al})$ phase grains for the given nucleation potential. In practice, the radical increase in the number of grains is grain refinement (also known as the modification process) (Ref 8,9). The benefits of grain refinement are improved fluidity, improved feeding, better distribution of porosity, and secondary phases, and moreover, it

M. Górny and G. Sikora, AGH University of Science and Technology, Reymonta Str. 23, 30-059 Krakow, Poland. Contact e-mail: mgorny@ agh.edu.pl.

improves surface quality, machinability, tensile properties, and pressure tightness (Ref 3 ). The grain refinement process increases the density of $\alpha(\mathrm{Al})$ phase nuclei as well as the amount of heat generated during solidification and, as a result, changes the degree of undercooling. It is also known that the effects of grain refinement disappear over time. The literature contains only limited data (Ref 10-13) about the relevant quantitative dependency of the cooling rate or the undercooling degree on the grain density of Al-Cu alloy.

The purpose of this study is to analyze the changes in the cooling rate of castings with different wall thicknesses and various titanium additions to demonstrate its significant impact on the primary grain count in castings and tensile properties of an Al-Cu alloy.

\section{Experimental Procedure}

As part of this research, Al-Cu alloy (201.2 according to ASTM B179-09) melts were prepared. The experimental melts were prepared in a medium-frequency induction furnace. The furnace charge consisted of the following charge materials: aluminum (purity: 99.85\%), AlCu50, AlMn75, and AlTi75. After the charge was melted, the covering and refining flux (1.2 wt.\%) was added onto the metal surface. The liquid metal having been heated to $750{ }^{\circ} \mathrm{C}$ was grain refined (alloy II and III), using the AlTi5B1 master alloy whose quantity was equal to $0.2 \%$ of the metal by weight. Alloys II and III differed in the amount of AlTi75 alloy additions in charge materials in order to obtain different titanium contents. After three minutes from the time of adding the AlTi5B1 master alloy, the liquid metal was cast into sets of molds made of a traditional molding sand with bentonite, forming standard type $\mathrm{Y}$ ingots (according to ASTM A536-84) with walls, respectively, 3, 5, 13, and $25 \mathrm{~mm}$ thick.

The results of the chemical composition analysis of tested alloys that was carried out using a SPECTRAMAXx emission spectrometer with spark excitation are shown in Table 1. 
In order to record cooling curves, 0.2-mm-thick tips of PtPtRh10 thermocouples were placed in the geometric centers of the bottom part of the ingots. An Agilent 34970A multi-channel electronic module was used to record temperature as the function of time. The temperature was recorded with a time step of $0.02 \mathrm{~s}$.

Figure 1 shows an example of a cooling curve (alloy II) together with its derivative $\mathrm{d} T / \mathrm{d} t$ as well as the method of determining the cooling rate $\dot{T}$ and the undercooling $\Delta T_{\alpha}$.

Samples for metallographic examinations were taken from the bottom part of the ingots and were then grinded, polished, and electrolytically etched, using Barker's reagent. During the etching, an electric current at $30 \mathrm{~V}$ was fed for the minimum time of $1 \mathrm{~min}$ in accordance with the ASTM E407-07 Standard.

Table 1 Results of chemical composition analyses of alloys I-III

\begin{tabular}{lllllll}
\hline & \multicolumn{6}{c}{ wt.\% } \\
\cline { 2 - 7 } Alloy No. & Si & Fe & Cu & Mn & B & Ti \\
\hline I & 0.05 & 0.11 & 4.90 & 0.40 & 0.0013 & 0.07 \\
II & 0.02 & 0.04 & 5.00 & 0.39 & 0.0021 & 0.11 \\
III & 0.04 & 0.03 & 4.15 & 0.33 & 0.0024 & 0.41 \\
\hline
\end{tabular}

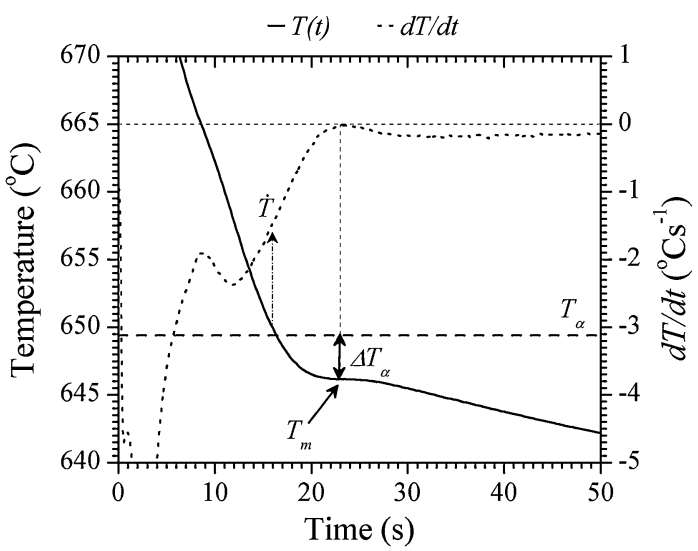

Fig. 1 Cooling curve $T(t)$ recorded in a casting with wall thickness of $13 \mathrm{~mm}$ and its first derivative $\mathrm{d} T / \mathrm{d} t$ for the alloy II

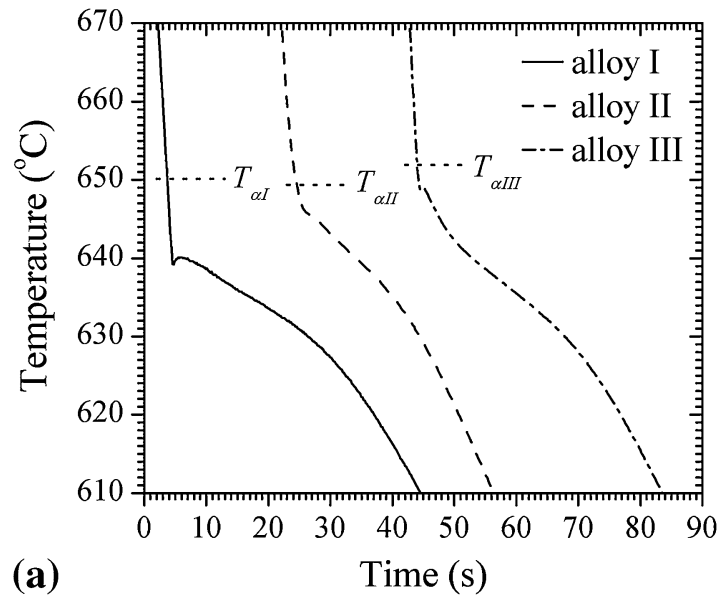

The metallographic examination was carried out with an optical stereomicroscope using polarized light.

The second option of the Jeffries method was used to determine the surface grain density $N_{\mathrm{A}}$, and, after applying Saltykov's formula, this density can be noted as (Ref 14):

$N_{\mathrm{A}}=\frac{N_{\mathrm{i}}+0.5 \times N_{\mathrm{c}}+1}{A}$,

where $N_{\mathrm{i}}$ is the number of grains contained in a rectangle with the surface area of $A . N_{\mathrm{c}}$ is the number of grains cut by the edges of the rectangle, excluding grains found in the corners. The final result was the arithmetical average of the grain density during the measurements of at least ten areas of the sample at a magnification of $20 \times$.

The volumetric density of grains $N_{\mathrm{v}}$ was calculated using the Voronoi relationship (Ref 15):

$N_{\mathrm{v}}=0.568 \times\left(N_{\mathrm{A}}\right)^{3 / 2}$.

Tensile testing (ultimate tensile strength, UTS and elongation, $s_{\mathrm{f}}$ ) was performed on flat samples in a universal Zwick/ Roell Z050, following the ASTM E8M 6.2 standard. In addition, samples were examined by a scanning electron microscope (SEM) operated at $20 \mathrm{kV}$.

\section{Results and Discussion}

\subsection{Thermal Analysis}

Figure 2 collates examples of cooling curves recorded in castings with walls 5 and $13 \mathrm{~mm}$ thick, respectively, for the analyzed alloys I-III.

The cooling rate $\dot{T}$ of castings was determined at the equilibrium alloy solidification temperature of phase $\alpha(\mathrm{Al})$. The undercooling value $\Delta T_{\alpha}$ was determined from the following relationship:

$\Delta T_{\alpha}=T_{\alpha}-T_{\mathrm{m}}$,

where $T_{\alpha}$ is the equilibrium temperature of the solidification of the $\alpha(\mathrm{Al})$ primary phase read from the $\mathrm{Al}-\mathrm{Cu}$ equilibrium system for the researched copper content; $T_{\mathrm{m}}$ is the minimum temperature at the beginning of the $\alpha(\mathrm{Al})$ phase solidification.

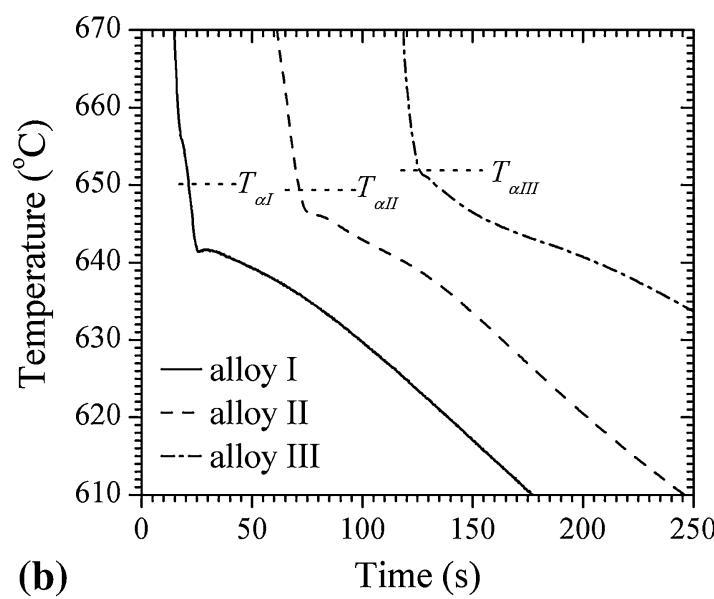

Fig. 2 Cooling curves of castings with wall thickness of 5 (a) and $13 \mathrm{~mm}$ (b) for alloys I-III 
Table 2 Results from thermal analysis

\begin{tabular}{|c|c|c|c|c|c|c|}
\hline \multirow[b]{2}{*}{$\mathrm{g}, \mathrm{mm}$} & \multicolumn{2}{|c|}{ Alloy I } & \multicolumn{2}{|c|}{ Alloy II } & \multicolumn{2}{|c|}{ Alloy III } \\
\hline & $\dot{T},{ }^{\circ} \mathrm{Cs}^{-1}$ & $\Delta T_{\infty}{ }^{\circ} \mathrm{C}$ & $\dot{T},{ }^{\circ} \mathrm{Cs}^{-1}$ & $\Delta T_{\infty},{ }^{\circ} \mathrm{C}$ & $\dot{T},{ }^{\circ} \mathrm{Cs}^{-1}$ & $\Delta T_{\infty}{ }^{\circ} \mathrm{C}$ \\
\hline 3 & 21.62 & 11.80 & 21.53 & 8.35 & 23.72 & 14.82 \\
\hline 5 & 12.79 & 10.93 & 11.29 & 3.90 & 12.03 & 3.09 \\
\hline 13 & 2.30 & 8.71 & 2.12 & 3.23 & 2.58 & 0.69 \\
\hline 25 & 1.17 & 8.26 & 1.61 & 0.20 & 1.67 & 1.46 \\
\hline
\end{tabular}

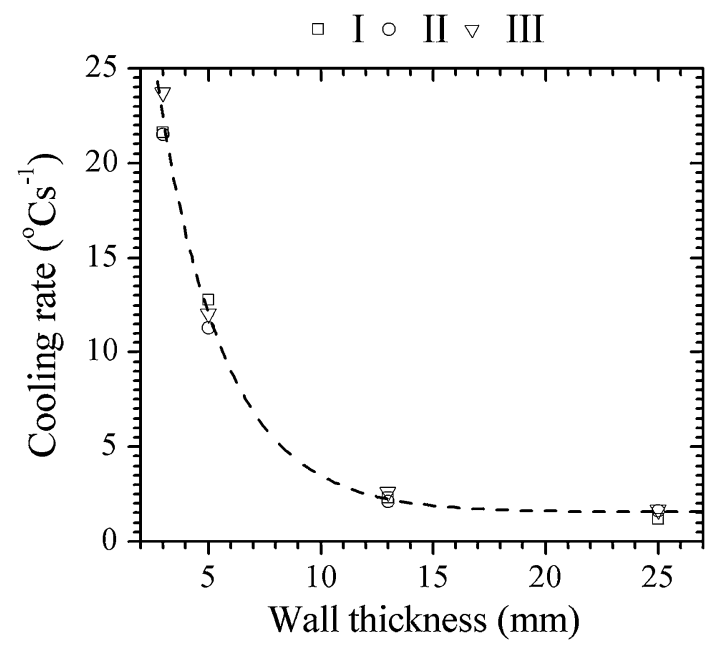

Fig. 3 The cooling rate as a function of the wall thickness of casting for the investigated alloys: experimental points for melts I-III and the regression curve- the dotted line

Table 2 presents the experimentally determined values of the cooling rate $\dot{T}$ and the maximum undercooling degree $\Delta T_{\alpha}$ for the obtained castings.

The dependency of the cooling rate as a function of the wall thickness of castings is graphically illustrated in Fig. 3.

The recorded cooling rate varies within a broad range (23.7-1.2 ${ }^{\circ} \mathrm{Cs}^{-1}$ ) when the wall thickness changes from 3 to $25 \mathrm{~mm}$ for all alloys. The cooling rate influences the maximum degree of undercooling and, as a result, also affects the structure of the examined alloys. If the cooling rate $\dot{T}$ rises, this increases the maximum degree of undercooling $\Delta T_{\alpha}$, contributing to an increase of the driving force of the solidification process. This is of particular importance for the refined alloys II and III, in which the degree of undercooling values are much lower than in the base alloy. It is worth mentioning that the increase in the degree of undercooling as a result of an increased cooling rate is much greater in the grainrefined alloys than in the base one.

The dependency of the cooling rate as a function of wall thickness can be approximated using an equation of the following form:

$Q=1.55+57.35 \times \exp (-0.339 \times g)$,

with a high correlation coefficient of $R=0.99$. This equation takes into account base alloy and grain-refined ones. The grain refinement process thus does not influence the cooling rate (Fig. 3) at the beginning of the $\mathrm{Al}-\mathrm{Cu}$ alloy solidification. This is also shown in the diagram presented in Fig. 4.
Figure 4 suggests that the cooling rate (the angle of the cooling rate to the time axis at the beginning of the solidification process) of the grain-refined alloy and the base alloy is the same. Liquid metal in both the grain-refined and base alloys has various nucleation potentials. This potential can be represented by a different number of nucleation sites of the $\alpha(\mathrm{Al})$ phase. Grain refinement causes millions of new nucleation sites to appear, which change the size distribution of sites in the heterogeneous nucleation (Fig. 4b). Increasing the efficiency of grain refinement by moving from the $n_{\mathrm{II}}(l)$ to $n_{\mathrm{III}}(l)$ size distribution of sites nucleation ensures a much greater number of nuclei at a lower level of undercooling (Fig. 4a). An equation identifying the number of $\alpha(\mathrm{Al})$ phase nuclei created at a maximum undercooling $\Delta T_{\alpha}$ can be presented based on the Weibull distribution of nucleation sites given by the equation of the following form (Ref 16):

$N_{\mathrm{v}}=n_{\mathrm{p}} \times \exp \left(-b / \Delta T_{\alpha}\right)$

where $n_{\mathrm{p}}$ is the number of all particles-substrates found in the liquid metal, $b$ is the nucleation coefficient which can be defined as follows:

$b=\frac{4 \sigma_{c, k}}{\Delta S \times l_{A}}$

where $\sigma_{\mathrm{c}, \mathrm{k}}$ is the surface energy at the nucleus-melt interface, $\Delta S$ is the entropy of solidification, $l_{\mathrm{A}}$ is the average nucleation site size.

Equation (5) describes the relationship between the maximum degree of undercooling and the nucleus count, and as it was mentioned before the grain count as well.

\subsection{Macroscopic Examinations}

Figure 5 shows examples of macrostructures portraying primary $\alpha(\mathrm{Al})$ phase grains in the tested alloys.

Calculated primary grain counts $N_{\mathrm{v}}$ and grain size $d$ for the base alloy and grain-refined alloys are presented in Table 3.

Figure 6 presents the relationship between the density of grains $N_{\mathrm{v}}$ as a function of the maximum degree of undercooling $\Delta T_{\alpha}$ for all investigated alloys.

It has already been mentioned that the cooling rate influences the maximum degree of undercooling, which, in turn, determines the number of nuclei, and thus of the grains (dendrites) of the $\alpha(\mathrm{Al})$ phase. This is shown in Fig. 6. Experimental data allow parameters $n_{\mathrm{p}}$ and $b$ from Eq. (5) to be determined. For the base alloy and grain-refined alloy, Eq. (5) takes the following form:

(a) For the base alloy (alloy I) $N_{\mathrm{v}}=131587 \times \exp (-25.39$ $\left./ \Delta T_{\alpha}\right)$ where $R=0.90$ 


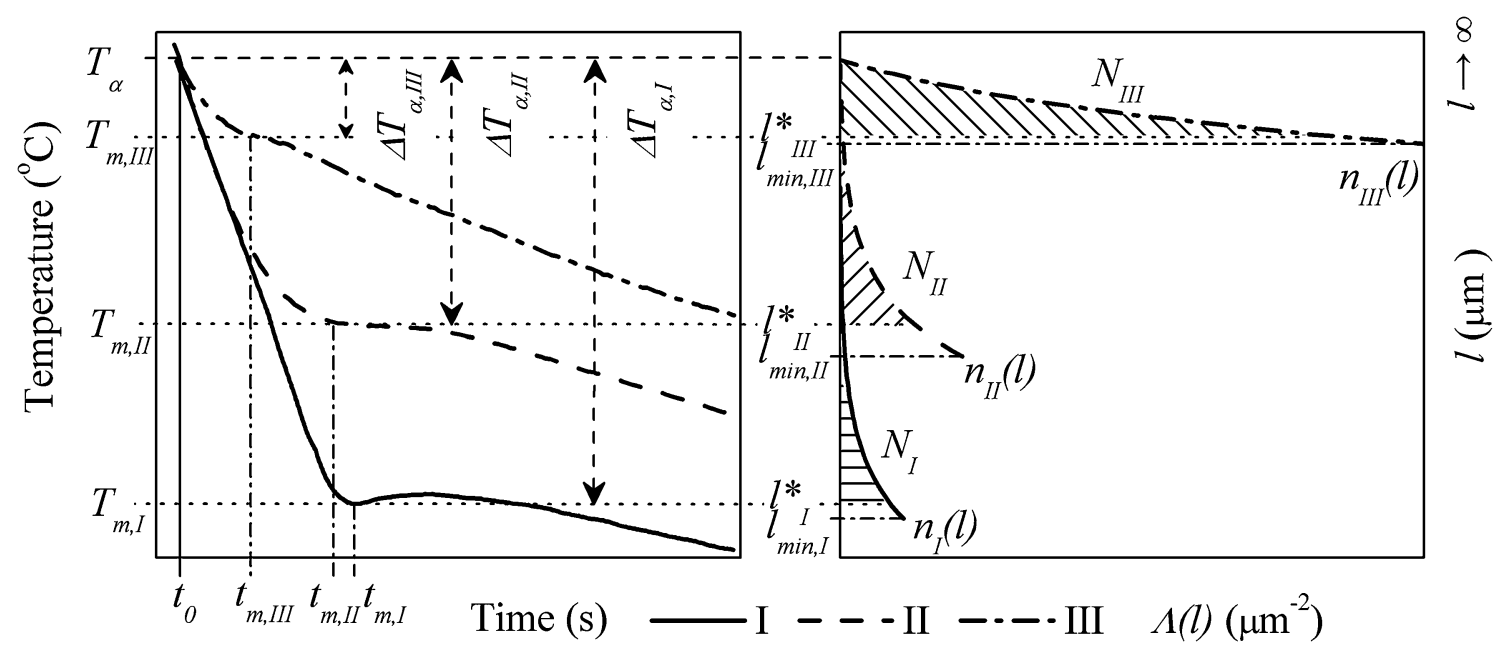

Fig. 4 Cooling curves $T_{\mathrm{I}}(t), T_{\mathrm{II}}(t)$, and $T_{\mathrm{II}}(t)$ for three nucleation potential of melt (a) and schematic representation of an $\alpha$-Al size distribution of nucleation sites (b)

(b) For the grain-refined (alloy $\times \exp \left(-4.68 / \Delta T_{\alpha}\right)$ where $R=0.93$

(c) For the grain-refined (alloy $\times \exp \left(-2.41 / \Delta T_{\alpha}\right)$ where $R=0.93$

II) $N_{\mathrm{v}}=365653$

III) $N_{\mathrm{v}}=778671$

Furthermore, it is apparent that the predictions of the analytical expressions (Eq. 5) are rather similar to those obtained from the experimental outcome (Fig. 6). Figure 6 also suggests that for a given nucleation potential, if the maximum degree of undercooling increases, so does the number of grains. In the case of castings with walls of the same thickness, but of various nucleation potential, if the maximum degree of undercooling goes up, the number of grains decreases. The transition from the base alloy through to the second one (grain refined-alloy II) to the third one (grain refined-alloy III) brings about a change in the density of nucleation sites $n_{\mathrm{p}}$ (from $n_{\mathrm{p}}=131,587 \mathrm{~cm}^{-3}$ through $n_{\mathrm{p}}=365,653 \mathrm{~cm}^{-3}$ to $\left.n_{\mathrm{p}}=778,671 \mathrm{~cm}^{-3}\right)$ and in the nucleation coefficient $b$ (from $b=25.39{ }^{\circ} \mathrm{C}$ through $b=4.68{ }^{\circ} \mathrm{C}$ to $b=2.41{ }^{\circ} \mathrm{C}$ ). The consequence of this, at a constant casting modulus (approximately at a constant cooling rate) is to reduce the density of $\alpha(\mathrm{Al})$ nucleus (grain density) and increase the degree of maximum undercooling $\Delta T_{\alpha}$ (see the arrows in Fig. 6).

The rise in the density of $\alpha(\mathrm{Al})$ nucleus (as a result of the grain refinement process) and thus also of the primary grains in $\mathrm{Al}-\mathrm{Cu}$ alloys results from an increase in the total number of heterogeneous nucleation sites caused by the formation of new particles in the liquid metal which determines the kinetics of the solidification. In temperature terms, it is reflected in the metal cooling curve. It should also be noted that if the nucleation potential had changed (as a result of grain refinement), the degree of undercooling $\Delta T_{\alpha}$ would have fallen, which is connected with an increase in the quantity of the solidification heat, generated by a greater number of primary grains of the $\alpha(\mathrm{Al})$ phase forming. A reduction of the degree of undercooling will be greater, the higher the nucleation potential of $\alpha(\mathrm{Al})$ phase, which is directly proportionate to the nucleus density $n_{\mathrm{p}}$ and the coefficient $b$ from Eq. (5). Please note that these parameters are strongly influenced by the entropy of solidification, surface tension, and average substrate site size. Consequently, they have a well-defined physical meaning in contrast with the empirical nucleation expressions which can be encountered in different computer modeling softwares (Ref 7). Due to the lack of relevant data (e.g., average site size, density of substrates, wetting angle) for the current state of knowledge, the values of $n_{\mathrm{p}}$ and $b$ parameters have to be estimated from experimental determinations of the number of grains and maximum undercooling.

\subsection{Tensile Properties}

The variation of the tensile properties of samples as a function of the cooling rate is presented in Fig. 7(a), while in Fig. 7(b), examples of stress-strain curves of tested alloy are shown. Experimental studies show that an increase in the cooling rate by reducing the wall thickness of castings causes substantial changes in tensile properties. In the case of alloy III, the highest values of ultimate tensile strength and elongation were achieved. Ultimate tensile strength and elongation values in a casting with a wall thickness of $25 \mathrm{~mm}\left(\dot{T}=1.4{ }^{\circ} \mathrm{Cs}^{-1}\right)$ are at a level of those obtained in the base alloy castings with a wall thickness of $3 \mathrm{~mm}\left(\dot{T}=23.7^{\circ} \mathrm{Cs}^{-1}\right)$.

It is worth noting that, in the grain-refined alloys with an increased cooling rate, the ultimate tensile strength differences are reduced, while elongation differences increase (Fig. 7a). It can therefore be concluded that the extensive primary grainrefining process, to a greater extent, is responsible for increasing the elongation of the alloy than for the growth of ultimate tensile strength (Fig. 8). From this figure, one can see that the UTS varies from 96 to $210 \mathrm{MPa}$, and the elongation to fracture increases from 1.5 to $14 \%$ when changing grain size from 695 to $148 \mu \mathrm{m}$.

This definitely suggests that the elongation to fracture is more sensitive to grain size value than the ultimate tensile strength.

From SEM examination results, the fracture surface of the base alloy is covered by smooth cleavage planes and minor dimples, suggesting that it belongs to a brittle fracture mode. For the grain-refined alloys, a rough surface and more dimples can be observed, especially in alloy III. 

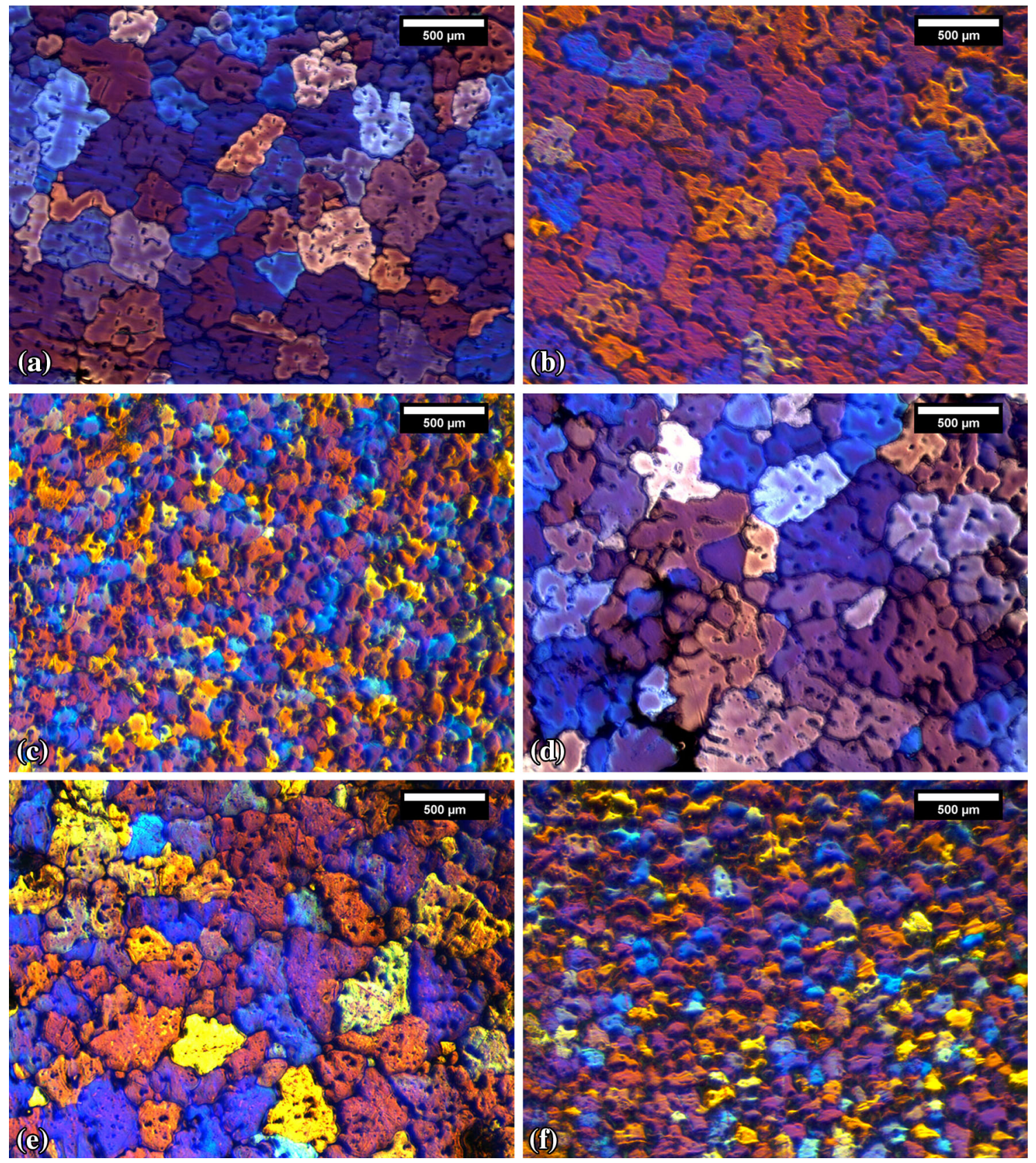

Fig. 5 Macrostructures of samples cut from castings: (a, d) base alloy: $g=13 \mathrm{~mm}$ and $g=25 \mathrm{~mm}$, respectively, (b, e) grain-refined alloy II: $g=13 \mathrm{~mm}$ and $g=25 \mathrm{~mm}$, respectively, (c, f) grain-refined alloy III: $g=13 \mathrm{~mm}$ and $g=25 \mathrm{~mm}$, respectively

Table 3 Volumetric density $N_{\mathrm{v}}$ and grain size $d$ of $\alpha(\mathrm{Al})$ primary grains as a function of the casting wall thickness $g$ for the tested alloys

\begin{tabular}{|c|c|c|c|c|c|c|}
\hline \multirow{2}{*}{$\begin{array}{l}\text { Melt no. } \\
g, \mathrm{~mm}\end{array}$} & \multicolumn{2}{|c|}{ Alloy I } & \multicolumn{2}{|c|}{ Alloy II } & \multicolumn{2}{|c|}{ Alloy III } \\
\hline & $N_{\mathrm{v}}, \mathbf{c m}^{-3}$ & $d, \mu \mathrm{m}$ & $N_{\mathrm{v}}, \mathbf{c m}^{-3}$ & $d, \mu \mathrm{m}$ & $N_{v}, \mathrm{~cm}^{-3}$ & $d, \mu \mathrm{m}$ \\
\hline 3 & 17.160 & 451 & 202.686 & 178 & 665.695 & 148 \\
\hline 5 & 10.094 & 493 & 145.123 & 256 & 365.703 & 157 \\
\hline 13 & 7.832 & 592 & 52.532 & 374 & 172.824 & 190 \\
\hline 25 & 6.503 & 695 & 19.613 & 525 & 88.110 & 217 \\
\hline
\end{tabular}


$\square$ I $\quad \circ$ II $\triangle$ III

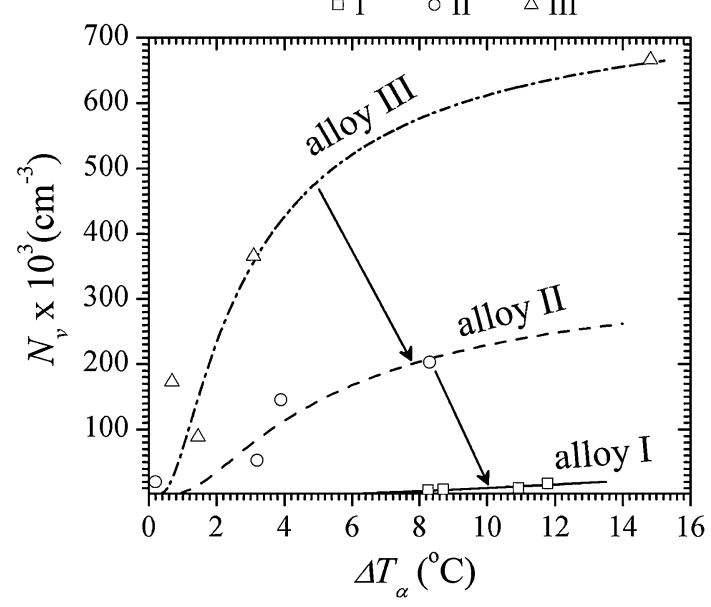

Fig. 6 Primary grain density as a function of the maximum degree of undercooling

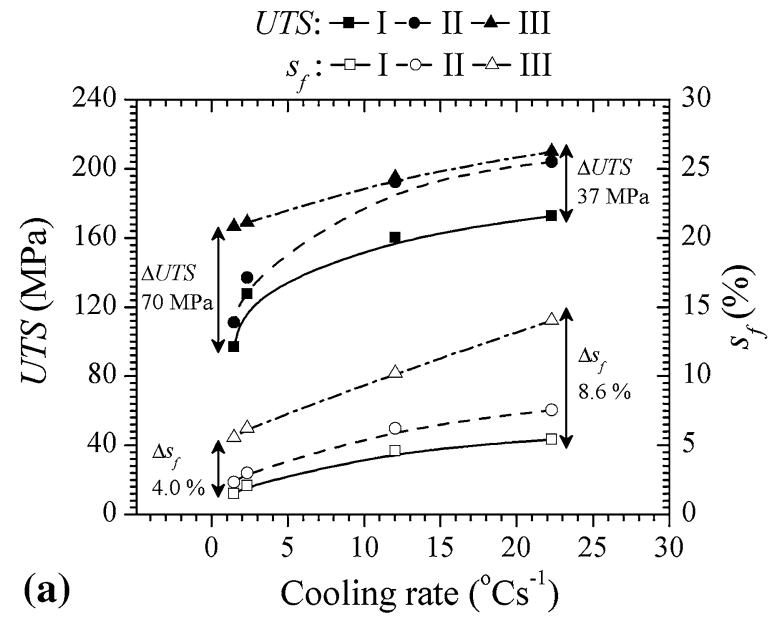

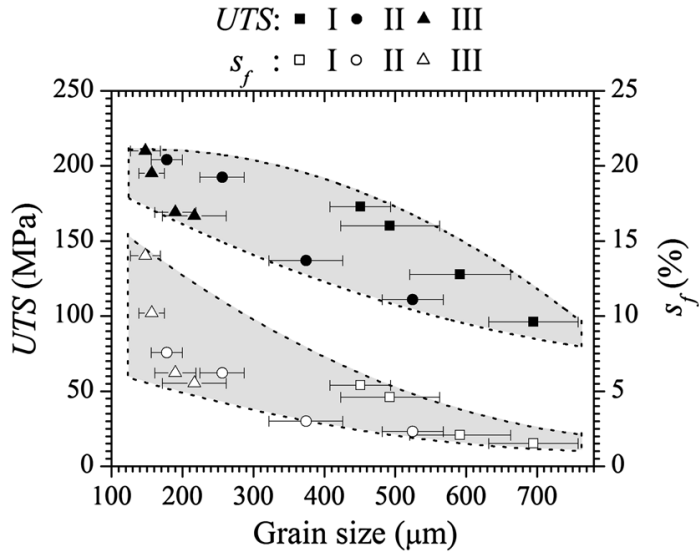

Fig. 8 UTS and elongation to fracture as function of grain size of the primary phase of $\alpha(\mathrm{Al})$

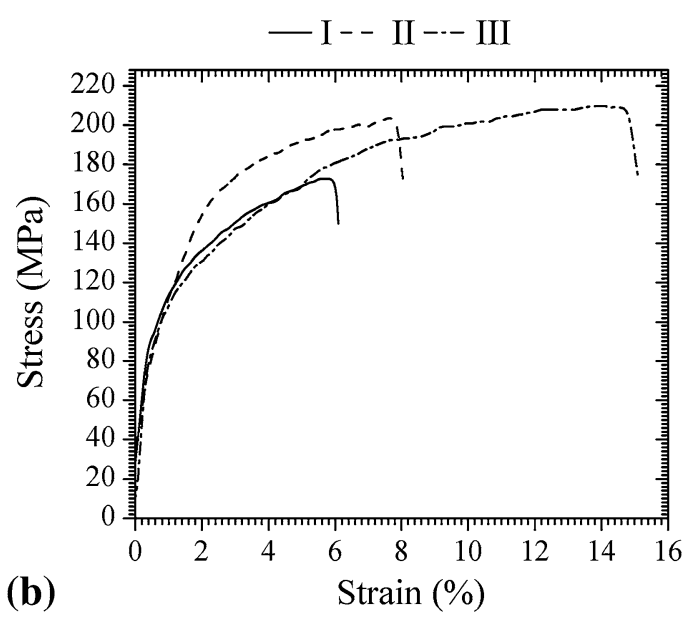

Fig. 7 Ultimate tensile strength UTS and elongation to fracture $s_{\mathrm{f}}$ as a function of cooling rate for tested alloys (a) and examples of stress-strain curves of tested alloy for castings with wall thickness of $3 \mathrm{~mm}$ (b)

\section{Conclusions}

1. Experimental research indicates that the connection between the density of primary grains $(\alpha(\mathrm{Al}))$ and the maximum degree of undercooling (for the base alloy and grain-refined alloys) can be noted with a high correlation coefficient in the form of a Weibull type expression (Eq. 5).

2. The cooling rate varies within a wide range (23.7-1.2 ${ }^{\circ} \mathrm{Cs}^{-1}$ ) when the wall thickness is changed from 3 to $25 \mathrm{~mm}$, and this is accompanied by a significant variation in the maximum undercooling. The grain refinement process does not influence the cooling rate at the beginning of solidification; however, it strongly influences the final number of $\alpha(\mathrm{Al})$ primary grains.

3. For the grain-refined alloys with an increase in the cooling rate caused by reducing the wall thickness of castings, the ultimate tensile strength differences are reduced, while elongation differences increase. Combining the cooling rate (from 1.4 to $23.7{ }^{\circ} \mathrm{Cs}^{-1}$ ) and the efficient Ti-based grain refiner increased the tensile ductility by up to $150 \%$.

\section{Open Access}

This article is distributed under the terms of the Creative Commons Attribution License which permits any use, distribution, and reproduction in any medium, provided the original author(s) and the source are credited.

\section{References}

1. G. Timelli, G. Camicia, and S. Farraro, Effect of Grain Refinement and Cooling Rate on the Microstructure and Mechanical Properties of Secondary Al-Si-Cu Alloys, J. Mater. Eng. Perform., 2014, 23, p 611-621

2. B.S. Murty, S.A. Kori, and M. Chakraborty, Grain Refinement of Aluminum and Its Alloys by Heterogeneous Nucleation and Alloying, Int. Mater. Rev., 2002, 47, p 3-29

3. J. Spittle, A Grain Refinement in Shape Casting of Aluminum Alloys, Int. J. Cast Met. Res., 2006, 19, p 210-222

4. R. Chen, Y. Shi, Q. Xu, and B. Liu, Effect of Cooling Rate on Solidification Parameters and Microstructure of Al-7Si-0.3Mg-0.15Fe Alloy, Trans. Nonferrous Met. Soc. China, 2014, 24, p 1645-1652

5. T.E. Quested and A.L. Greer, Grain Refinement of Al Alloys: Mechanisms Determining As-Cast Grain Size in Directional Solidification, Acta Mater., 2005, 53, p 4643-4653 
6. T.E. Quested and A.L. Greer, The Effect of the Size Distribution of Inoculant Particles on As-Cast Grain Size in Aluminum Alloys, Acta Mater., 2004, 52, p 3859-3868

7. D. M. Stefanescu, Science and Engineering of Casting Solidification, Kulwer/Plenum, New York, 2002

8. H. Xiaowu, A. Fanrong, and Y. Hong, Influence of Pouring Temperature and Cooling Rate on Microstructure and Mechanical Properties of Casting Al-Si-Cu Aluminum Alloy, Acta Metall. Mater, 2012, 25, p $272-278$

9. F. Grosselle, G. Timelli, F. Bonollo, A. Tiziani, and E. Della Corte, Correlation Between Microstructure and Mechanical Properties of AlSi Cast Alloy, Metall. Ital., 2009, 101, p 25-32

10. M.A. Easton and D.H. Stjohn, The Effect of Grain Refinement on the Formation of Casting Defects in Alloy 356 Castings, Int. J. Cast Met. Res., 2000, 12, p 393-408
11. S. Lin, C. Aliravci, and M.O. Pekguleryuz, Hot-Tear Susceptibility of Aluminum Wrought Alloys and The Effect of Grain Refining, Metall. Mater. Trans. A, 2007, 38, p 1056-1068

12. D. Eskin, Q. Du, and D. Ruvalcaba, Experimental Study of Structure Formation in Binary Al-Cu Alloys at Different Cooling Rates, Mater. Sci. Eng. A, 2005, 404, p 1-10

13. L. Bäckerud, Solidification Characteristics of Aluminium Alloys, Skanaluminium, Oslo, 1986

14. J. Rys, Stereology of Materials, Fotobit, 1995 (Cracow)

15. J. Ohser, U. Lorz, Quantitative Gefuegeanalyse: theoretische Grundlagen und Anwendung, DVG, 1994 (Leipzig-Stuttgart)

16. E. Fras, K. Wiencek, M. Gorny, H.F. Lopez, and E. Olenik, Equiaxed Grain Count in Aluminum Alloy Castings: Theoretical Background and Experimental Verification, Metall. Mater. Trans. A, 2013, 44, p 57885795 